\title{
Analytical Solutions in the Framework of Brightness and Color Spectral Pyrometry Methods
}

\author{
Konstantin Ludanov \\ Department No 5, Institute for Renewable Energy of NASU, Kiev, Ukraine
}

Email address:

k.i.ludanov@ukr.net

To cite this article:

Konstantin Ludanov. Analytical Solutions in the Framework of Brightness and Color Spectral Pyrometry Methods. World Journal of Applied Physics. Vol. 4, No. 3, 2019, pp. 35-40. doi: 10.11648/j.wjap.20190403.11

Received: July 18, 2019; Accepted: August 22, 2019; Published: September 27, 2019

\begin{abstract}
The article provides an analytical solution to the problem of pyrometric determination of the actual surface temperature $T(K)$ by calculating its value from a system of three equations, including the results of three measurements of the brightness temperature $T_{S}\left(\lambda_{i}\right)$, which were obtained at three wave length sof the spectrum: $\lambda_{1}<\lambda_{2}<\lambda_{3}$ with unknown emissivity $\varepsilon_{1}, \varepsilon_{2}$ and $\varepsilon_{3}$, in the framework of the two main methods of spectral pyrometry according to the patents of Ukraine No.73231 (color) and No.82870 (brightness). It is shown in the article that exact analytical solutions are possible only in cases when the nature of the dispersion of the selective surface $\varepsilon=\mathrm{f}(\lambda)$ (for example, linear, logarithmic, etc.) is known apriori, which, together with the equation of coupling $\varepsilon_{2}=\mathrm{F}\left(\varepsilon_{1}, \varepsilon_{3}\right)$, allows us to determine the exact value of the intermediate (effective) length $\lambda_{2}$ waves based on the extreme (or basic) values of $\lambda_{1}$ and $\lambda_{3}$. The arithmetic mean (for the color method) and the proportional mean (for the brightness method) are use dascoupling equations.
\end{abstract}

Keywords: Spectral Pyrometry, Brightness Pyrometer, Color Pyrometer, Double Spectral Ratio, Trichromatic Method, Coupling Equation

\section{Introduction}

In modern thermometry, the task of measuring surface temperatures in cases where it is so high that it is no longer possible to use thermocouples is very important. In such cases, use pyrometers. Pyrometers can be used for measurements and at low temperatures, but only in those cases where thermocouples cannot be placed on the surface (for example, for surfaces of revolution, etc.). And also in cases of determining rapidly changing surface temperatures in non-stationary processes, since thermocouples in such cases give a very high error, since they have a large inertia (time constant $\tau_{\mathrm{o}}$ ).

Pyrometry is a contactless thermometry method in which the absolute temperature $\mathrm{T}(\mathrm{K})$ of a certain surface is determined on the basis of measurements of the intensity of its own thermal radiation. However, most traditional methods of pyrometric temperature measurement have one fundamental flaw: the result of their measurement is not the actual temperature, but the "pseudo-temperature" ("radiation", "brightness" or "color"), for converting which into actual temperature it is necessary to know a priori the radiative temperature surface abilities. Most often such data is missing or unreliable. Therefore, usually when pyrometric measurement of the temperature of any surface, it is necessary to additionally determine its emissivity $\varepsilon(\lambda, T)$. Existing methods for measuring emissivity are very complex and expensive, so the task of developing combined methods of spectral pyrometry in which three or more pseudotemperature measurements are taken in different spectral ranges is very urgent, and then combining the equations obtained so that the result of determining $\mathrm{T}(\mathrm{K})$ did not depend on the emissivity of the surface.

\section{Literature Review}

The theoretical basis of radiation pyrometry was the Stefan-Boltzmann law $\left(E=\sigma_{0} \mathrm{~T}^{4}\right)$, established empirically by Stephen in 1879 and theoretically substantiated by Boltzmann in $1884[1,2]$. However, the first pyrometer, invented by Le Chatelier in 1892, was based not on integral, but on spectral regularities of thermal radiation. Although the Wien's formula [4], which is used as a theoretical basis for spectral pyrometers, was established a little later, in 1896, the 
use of an Le Chatelier optical pyrometer showed that spectral pyrometry due to measurements then made it possible to get an unreal value of the absolute surface temperature, but only a certain estimate of it, that is, a conditional "pseudotemperature", for recalculating it into temperature $\mathrm{T}(\mathrm{K})$, a preliminary determination of its emissivity $\varepsilon$ is necessary. The first attempt to solve this problem without determining $\varepsilon$ was the introduction of an additional equation (for the closure of a system of equations) in the form of a dispersion curve of the radiating power $\varepsilon=\mathrm{f}(\lambda)$ of a flame (according to Bouguer law). It was first used by Angstrom in 1889 when estimating the temperature of the flame of a stearin candle.

Regarding the choice between methods of spectral and radiation pyrometry [3-5], it can be said that radiation method is not suitable for measuring the temperature of surfaces for which its changes very rapidly with time, which happens in non-stationary processes of cooling or heating. In such cases, only spectral methods make it possible to obtain most accurate values of $\mathrm{T}(\tau)$, since their "time constant" $\tau_{\mathrm{o}}$ is very small (time constant $\tau$ o of photodiodes is $10^{-6}-10^{-7}$ sec.).

The method of brightness pyrometry [6] is widely known for determining the temperature $\mathrm{T}$ of a selectively radiating surface. Typically, in this method, the brightness temperature $\mathrm{T}_{\mathrm{S}}$ is determined by the formula: $\left(\mathrm{T}_{\mathrm{S}}\right)^{-1}=\mathrm{T}^{-1}-\left(\lambda / \mathrm{C}_{2}\right) \cdot \ln (\varepsilon)$ using the known emissivity of the surface (usually measured for $\lambda=0.65 \mu \mathrm{m}, \mathrm{C}_{2}=1.439 \cdot 10^{4} \mu \mathrm{m} \cdot \mathrm{K}$ ). Its values are taken from the relevant section of the pyrometry reference books [6]. The databank presented in the reference books is obtained from a formula for $\mathrm{T}_{\mathrm{S}}$ by measuring the brightness temperature of surfaces whose temperature is known. The main disadvantage of this method is that in most cases the value of the spectral emittance of surfaces $\varepsilon$ given in reference books was previously determined at any one specific temperature. And it is known that the emissivity of materials significantly depends on temperature, for example, for metals, using the Ashkinass formula: $\varepsilon=5.67 \mathrm{~V}\left(\mathrm{~T} \cdot \mathrm{r}_{\mathrm{o}}\right)$ [7], where $r_{o}$ is the electrical resistivity. In addition, in real cases, the value of $\varepsilon$ depends on the surface treatment, the presence of oxides on it, etc. And the use of data from the directory without taking into account their influence on the result leads to significant errors in determining T. Moreover, in many cases, the values of the spectral emissivity surfaces are unknown even at $\lambda=0.65 \mu \mathrm{m}$.

Sometimes in the case when the values of the spectral emissivity are unknown a priori, a pair of brightness temperature measurements at different wavelengths of $\lambda_{1}<\lambda_{2}$ are used for pyrometric estimation of the surface temperature. This method is called the method of double spectral ratio or color method. To implement it, that is, determining the color pseudo-temperature of $\mathrm{T}_{\mathrm{C} 12}$, use the results of measuring two brightness temperatures $\mathrm{T}_{\mathrm{Si}}$ under the condition $\varepsilon_{1} \approx \varepsilon_{2}$, and based on them, get the color temperature of $\mathrm{T}_{\mathrm{C} 12}$ using the formula $\left(\mathrm{T}_{\mathrm{C} 12}\right)^{-1}=\mathrm{T}^{-1}$ $\left(\Lambda_{12} / C_{2}\right) \cdot \ln \left(\varepsilon_{1} / \varepsilon_{2}\right)$, where $\left(\Lambda_{12}\right)^{-1}=\left(\lambda_{1}\right)^{-1}-\left(\lambda_{2}\right)^{-1},\left(\lambda_{1}<\lambda_{2}\right)$ [8]. It is established that the color temperature gives a more accurate estimate of the true $\mathrm{T}$ than the brightness $\mathrm{T}_{\mathrm{S}}$. And to determine the true surface temperature $\mathrm{T}$ in the framework of this method, it is necessary to have a ratio between $\varepsilon_{1}$ and $\varepsilon_{2}$ (in the form $\varepsilon_{1}=\mathrm{k} \cdot \varepsilon_{2}$ for A. Magunov [9]). But the analysis shows that this is a special and very rare case, since the value of $\mathrm{k}$ is unknown.

In 1954, the English scientist E. Payet [10] for the first time solved the problem of pyrometric determination of the true temperature $\mathrm{T}$ of a selective surface without using information about the absolute values of its emissivity at all. For this, he used the method of trichromatic brightness pyrometry. At the same time he had to postulate that the expression of the dispersion $\varepsilon(\lambda)$ of the emissivity of the surface is linear: $\varepsilon(\lambda)=a+b \lambda$. The solution to this problem was obtained by determining the three brightness temperatures $\mathrm{T}_{\mathrm{S} 1}, \mathrm{~T}_{\mathrm{S} 2}$ and $\mathrm{T}_{\mathrm{S} 3}:\left(\mathrm{T}_{\mathrm{Si}}\right)^{-1}=\mathrm{T}^{-1}-\left(\lambda_{\mathrm{i}} / \mathrm{C}_{2}\right) \cdot \ln \left(\varepsilon_{\mathrm{i}}\right)$ for three different wavelengths $\lambda_{1}<\lambda_{2}<\lambda_{3}$, and in this case it was assumed that the intermediate emissivity of the surface $\varepsilon_{2}$ is equal to the arithmetic average of two extreme values: $\varepsilon_{2}=$ $\left(\varepsilon_{1}+\varepsilon_{3}\right) / 2$. That is, here the connection between $\varepsilon_{1}, \varepsilon_{2}$ and $\varepsilon_{3}$ was used for the first time, which allowed eliminating $\varepsilon_{1}, \varepsilon_{2}$ and $\varepsilon_{3}$ from the final expression for the true temperature in the solution process and getting the equation from only one unknown $\mathrm{T}$, and the last is to solve by numerical method.

The method of "double spectral ratio" is also known (this is the second modification of the method of trichromatic pyrometry), which is used to determine the true temperature $\mathrm{T}$ of the selectively radiating surface in the case when the logarithm of the surface spectral emitting power $\varepsilon(\lambda)$ linearly depends on the wavelength: $\ln \varepsilon(\lambda)=a+b \lambda$ [11]. This method also first measures three brightness pseudotemperatures $\mathrm{T}_{\mathrm{Si}}$ for three wavelengths $\lambda_{1}, \lambda_{2}$ and $\lambda_{3}$, respectively, and on this basis write a system of two equations for two spectral ratios (each of which is an expression for the color pseudo-temperature $\left(\mathrm{T}_{\mathrm{Cij}}\right)^{-1}=\mathrm{T}^{-1}$ $\left(\Lambda_{\mathrm{ij}} / \mathrm{C}_{2}\right) \cdot \ln \left(\varepsilon_{\mathrm{i}} / \varepsilon_{\mathrm{j}}\right)$. Based on the three results of determining the $\mathrm{T}_{\mathrm{Si}}$, it is possible to calculate the true temperature of the surface $\mathrm{T}$ by solving a system of two transcendental equations [7]. The main disadvantage of this method is that since the system of transcendental equations cannot be solved in general, the true surface temperature $\mathrm{T}$ can only be found as a result of a numerical solution of the system.

\section{Formulation of the Problem}

Thus, two methods of spectral pyrometry to determine the true temperature of the selective surface in the framework of trichromatic pyrometry are already known. The first is a combination of three measurements of $T_{S}$ brightness temperatures for the case of linear dispersion of surface emissivity: $\varepsilon=a+b \lambda$. In this case, it is assumed that the intermediate emissivity of the surface $\varepsilon_{2}$ is equal to the arithmetic average of $\varepsilon_{1}$ and $\varepsilon_{3}: \varepsilon_{2}=\left(\varepsilon_{1}+\varepsilon_{3}\right) / 2$, which is used as the "coupling equation".

The mentioned publication of E. Payet [10] is a very important, pioneer result for spectral pyrometry. However, it must be admitted that it is not a general solution, but only a special case, and one that can be realized only for surfaces with a linear nature of the dispersion of its emissivity.

The second case (D. Svet, 1955), i.e. the double spectral 
ratio method is a combination of two color methods [11] also for the case of linear dispersion, but already for the logarithm of the emissivity of the surface from the wavelength: $\ln \varepsilon(\lambda)=$ $a+b \lambda$. This result, by the way, is also a special case, not a general solution.

An analysis of both cases shows that in spectral pyrometry, the use of "coupling equations" makes it possible to solve a system of three equations (representing the results of three pyrometric measurements of the brightness temperature at three wavelengths $\left(\lambda_{1}, \lambda_{2}\right.$ and $\left.\lambda_{3}\right)$, for both spectral methods: and the trichromatic brightness method, and combinations of two color methods. The possibility of such a "closure" in the process of solving the system of equations unknowns $\varepsilon_{1}, \varepsilon_{2}$ and $\varepsilon_{3}$, while there is only one unknown - the absolute temperature T. But it should be noted that, first, so far only separate special cases for the dispersion expressions $\varepsilon=f(\lambda)$ of the emissivity of the surface, and secondly, the determination of the final result in them was possible only by numerical solution of the system of equations.

Therefore, the purpose of this study is to find an analytical solution to the problem, by definition, within the framework of trichromatic pyrometry, the actual surface temperature in an explicit form, and for any given dispersion of the emissivity of this surface.

\section{Results of Research}

\subsection{Combined Color Pyrometry Method}

July 14, 2003 author of the article filed a patent application with Ukrpatent for a new technical solution "Combined method of trichromatic color pyrometry". This invention [12], relates to the field of spectral pyrometry and can be used in determining the actual temperature $\mathrm{T}(\mathrm{K})$ by a color pyrometry of a selectively radiating surface with any known dispersion pattern $\varepsilon=f(\lambda)$ in the working range of the pyrometer, but only under the condition $\varepsilon_{2}=\left(\varepsilon_{1}+\varepsilon_{3}\right) / 2$.

In this case, the logarithm of the ratio $\varepsilon_{1} / \varepsilon_{3}$ from $\left(\mathrm{T}_{\mathrm{Cij}}\right)^{-1}=$ $\mathrm{T}^{-1}-\left(\Lambda_{\mathrm{ij}} / \mathrm{C}_{2}\right) \cdot \ln \left(\varepsilon_{\mathrm{i}} / \varepsilon_{\mathrm{j}}\right)$ can be expressed through the logarithms $\varepsilon_{1} / \varepsilon_{2}$ and $\varepsilon_{2} / \varepsilon_{3}$ as follows:

$$
\ln \left(\varepsilon_{1} / \varepsilon_{3}\right)=\ln \left[\left(\varepsilon_{\mathrm{AR}}+\Delta \varepsilon / 2\right) /\left(\varepsilon_{\mathrm{AR}}+\Delta \varepsilon / 2\right)\right]=2 \operatorname{arth}\left(\Delta \varepsilon / 2 \varepsilon_{\mathrm{AR}}\right),(1)
$$

where $\Delta \varepsilon=\varepsilon_{1}-\varepsilon_{3}, \Delta \varepsilon / 2 \varepsilon_{\mathrm{AR}}=\left(\varepsilon_{1}-\varepsilon_{3}\right) / 2 \varepsilon_{2}=\left(\varepsilon_{1} / \varepsilon_{2}-\varepsilon_{3} / \varepsilon_{2}\right) / 2$.

As a result of solving the problem, a closed solution is obtained for one unknown, but the expression for $\mathrm{T}$ was obtained in an implicit form:

$$
\begin{aligned}
\mathrm{T}^{-1}= & \mathrm{T}_{\mathrm{C} 13}^{-1}-2\left(\Lambda_{13} / \mathrm{C}_{2}\right) \cdot \operatorname{arth}\left[( 1 / 2 ) \left\{\exp \left[\left(\mathrm{C}_{2} / \Lambda_{12}\right)\left(\mathrm{T}_{\mathrm{C} 12}^{-1}-\mathrm{T}^{-1}\right)\right]-\right.\right. \\
& \left.\left.\exp \left[\left(\mathrm{C}_{2} / \Lambda_{23}\right)\left(\mathrm{T}_{\mathrm{C} 23}^{-1}-\mathrm{T}^{-1}\right)\right]\right\}\right],
\end{aligned}
$$

where $\mathrm{T}_{\mathrm{Cij}}=\left(\Lambda_{\mathrm{ij}}\right)^{-1} /\left[\left(\lambda_{\mathrm{i}} \mathrm{T}_{\mathrm{Si}}\right)^{-1}-\left(\lambda_{\mathrm{j}} \mathrm{T}_{\mathrm{Sj}}\right)^{-1}\right],\left(\Lambda_{\mathrm{ij}}\right)^{-1}=\left(\lambda_{\mathrm{i}}\right)^{-1}-\left(\lambda_{\mathrm{j}}\right)^{-1}$.

Therefore, the determination of the exact value of $\mathrm{T}$ from this implicit expression can be carried out by the method of successive approximations [13], that is, by setting the value of the first approximation $\mathrm{T}_{\mathrm{i}=1}$ and carrying out a series of successive approximations (iterations) using formula $\mathrm{T}_{\mathrm{i}+1}=$ $\mathrm{F}\left(\mathrm{T}_{\mathrm{i}}, \mathrm{T}_{\mathrm{C} 12}, \mathrm{~T}_{\mathrm{C} 23}, \mathrm{~T}_{\mathrm{C} 13}\right)$ :

$$
\begin{aligned}
\mathrm{T}^{-1}{ }_{\mathrm{i}+1}= & \mathrm{T}_{\mathrm{C} 13}^{-1}-2\left(\Lambda_{13} / \mathrm{C}_{2}\right) \cdot \operatorname{arth}\left[( 1 / 2 ) \left\{\exp \left[\left(\mathrm{C}_{2} / \Lambda_{12}\right)\left(\mathrm{T}_{\mathrm{C} 12}^{-1}-\mathrm{T}_{\mathrm{i}}^{-1}\right)\right]-\right.\right. \\
& \left.\left.\exp \left[\left(\mathrm{C}_{2} / \Lambda_{23}\right)\left(\mathrm{T}^{-1}{ }_{\mathrm{C} 23}-\mathrm{T}_{\mathrm{i}}^{-1}\right)\right]\right\}\right] .
\end{aligned}
$$

Moreover, iterations are carried out until the value of the difference between the results of the last and the penultimate approximations is less than the experimental error. The result of the last approximation is the desired $\mathrm{T}$ value for the actual surface temperature.

\subsection{Definitions of Intermediate (Effective) Wavelength $\lambda_{2}$ for Color Pyrometry}

However, in order to obtain all the results given above, it is necessary to choose the values of the intermediate wavelength $\lambda_{2}$ in such a way as to satisfy the equality $\varepsilon_{2}=$ $\left(\varepsilon_{1}+\varepsilon_{3}\right) / 2$, using the concept of the "inverse" function $\lambda=$ $\mathrm{f}^{-1}(\varepsilon)$ for the dispersion of the spectral emissivity in the working range of the pyrometer. For a selectively radiating surface with a known character of the dependence of the spectral emissivity on the wavelength $\varepsilon=f(\lambda)$, preliminarily determine the value of the intermediate wavelength $\lambda$ by the formula: $\lambda_{2}=\mathrm{f}^{-1}\left(\varepsilon_{2}\right) \equiv \mathrm{F}\left(\varepsilon_{\mathrm{AR}}\right)$, where $\lambda=\mathrm{f}^{-1}(\varepsilon)$ is the inverse function given for $\varepsilon=\mathrm{f}(\lambda), \varepsilon_{\mathrm{AR}}$ is the arithmetic average of the extreme values $\varepsilon_{1}$ and $\varepsilon_{3}$.

As it turned out, the value of the intermediate wavelength depends only on the base wavelengths $\lambda_{1}$ and $\lambda_{3}$, and does not depend on the absolute values of the spectral emissivity $\varepsilon_{1}, \varepsilon_{2}$ and $\varepsilon_{3}$. In cases when the base values for the extreme wavelengths of the working range $\left(\lambda_{1} \div \lambda_{3}\right)$ are selected based on the minimum instrumental error (for example, for a color pyrometer from a "fading thread", where $\lambda_{1}=0.43 \mu \mathrm{m}, \lambda_{3}=$ $0.65 \mu \mathrm{m}$ is historically the first "blue-red" ratio of wavelengths $\lambda_{1}$ and $\lambda_{3}$, which are the borders of the "sensitivity window" of the human eye), then the value of $\lambda_{2}$ is completely determined by the dispersion of the spectral emissivity $\varepsilon=f(\lambda)$ of the selectively radiating surface.

In those cases when "transparency windows" in the atmosphere air are more important, choose different wavelengths $\lambda_{1}$ and $\lambda_{3}$.

EXAMPLE 1.

If the dependence character for the dispersion $\varepsilon=f(\lambda)$ of the radiating surface has the linear expression $\varepsilon=a+b \lambda$, then the inverse function for $\varepsilon=\mathrm{f}(\lambda)$ has the following form: $\lambda=(\varepsilon-a) / b$. Then $\lambda_{2}=\left(\varepsilon_{2}-a\right) / b$. Substituting instead of $\varepsilon_{2}$ its value from the equation of communication $\varepsilon_{2}=\left(\varepsilon_{1}+\varepsilon_{3}\right) / 2$, we get:

$$
\begin{aligned}
\lambda_{2}=\left[\left(\varepsilon_{1}+\varepsilon_{3}\right) / 2-a\right] / b= & \left\{\left[\left(a+b \lambda_{1}\right)+\left(a+b \lambda_{3}\right)\right] / 2-a\right\} / b= \\
& \left(\lambda_{1}+\lambda_{3}\right) / 2 .
\end{aligned}
$$

Thus, if it is known a priori that the dispersion pattern of the surface spectral $\varepsilon=f(\lambda)$ in the working range of the pyrometer has a linear form: $\varepsilon=a+b \lambda$, then the brightness temperature $\mathrm{T}_{\mathrm{S}}\left(\lambda_{2}\right)$ should be measured at an intermediate wavelength $\lambda_{2}\left(\lambda_{1}<\lambda_{2}<\lambda_{3}\right)$, which is equal to the arithmetic average of the base wavelengths at the borders of the working range:

$$
\lambda_{2}=\left(\lambda_{1}+\lambda_{3}\right) / 2
$$


For the case of $\lambda_{1}=0.43 \mu \mathrm{m}, \lambda_{3}=0.65 \mu \mathrm{m}$, we get $\lambda_{2}=$ $0.540 \mu \mathrm{m}$.

\section{EXAMPLE 2.}

Since the dependence character $\varepsilon=\mathrm{f}(\lambda)$ for metals (Drude formula) has the form $\varepsilon=0.365 \sqrt{ }(\mathrm{r} / \lambda)$, the inverse function for $\varepsilon=\mathrm{f}(\lambda)$ is as follows: $\lambda=\mathrm{r} \cdot(0.365 / \varepsilon)^{2}$. Then $\lambda_{2}=$ $\mathrm{r} \cdot\left(0.365 / \varepsilon_{2}\right)^{2}$. Substituting instead of $\varepsilon_{2}$ its value from the

$$
\begin{gathered}
\lambda_{2}=0.365^{2} \cdot \mathrm{r} /\left[\left(\varepsilon_{1}+\boldsymbol{\varepsilon}_{3}\right) / 2\right]^{2}=4 \cdot 0.365^{2} \cdot \mathrm{r} /\left[0,365 \sqrt{ }\left(\mathrm{r} / \lambda_{1}\right)\right. \\
\left.+0.365 \sqrt{ }\left(\mathrm{r} / \lambda_{3}\right)\right]^{2}=4 /\left[\sqrt{ }\left(\lambda_{1}\right)^{-1}+\sqrt{ }\left(\lambda_{3}\right)^{-1}\right]^{2} .
\end{gathered}
$$

Since it is known a priori that for a metal surface the dispersion pattern of the spectral emissivity $\varepsilon=f(\lambda)$ in the working range of the pyrometer has the form $\varepsilon=0.365 \sqrt{ }(\mathrm{r} / \lambda)$, the brightness temperature $\mathrm{T}_{\mathrm{S}}\left(\lambda_{2}\right)$ must be measured at an intermediate wavelength $\lambda_{2}\left(\lambda_{1}<\lambda_{2}<\lambda_{3}\right)$, which is also equal to no one "average" from the base wavelengths at the equality $\sqrt{\varepsilon_{1} \cdot \varepsilon_{3}}$. This condition can be met mathematically rigorously - using the concept of the "inverse" function for $\lambda(\varepsilon): \lambda_{2}=\mathrm{f}^{-1}\left(\varepsilon_{2}\right)=\mathrm{f}^{-1}\left(\sqrt{\varepsilon_{1} \cdot \varepsilon_{3}}\right) \equiv \mathrm{F}\left(\sqrt{f\left(\lambda_{1}\right) \cdot f\left(\lambda_{3}\right)}\right)$, i.e. for dispersion of spectral emissivity in the working range of the wave pyrometer $\lambda_{2}\left(\lambda_{1}<\lambda_{2}<\lambda_{3}\right)$.

Since it is known a priori that for a metal surface the dispersion pattern of the spectral emissivity $\varepsilon=f(\lambda)$ in the working range of the pyrometer has the form $\varepsilon=0.365 \sqrt{ }(\mathrm{r} / \lambda)$, the brightness temperature $\mathrm{T}_{\mathrm{S}}\left(\lambda_{2}\right)$ must be measured at an intermediate wavelength $\lambda_{2}\left(\lambda_{1}<\lambda_{2}<\lambda_{3}\right)$, which is also equal to no one "average" from the base wavelengths at the boundaries of the working range of the pyrometer:

$$
\sqrt{ }\left(\lambda_{2}\right)^{-1}=\left[\sqrt{ }\left(\lambda_{1}\right)^{-1}+\sqrt{ }\left(\lambda_{3}\right)^{-1}\right] / 2 .
$$

For the case of $\lambda_{1}=0.43 \mu \mathrm{m}, \lambda_{3}=0.65 \mu \mathrm{m}$, we get $\lambda_{2}=$ $0.523 \mu \mathrm{m}$.

\subsection{Combined Brightness Pyrometry Method}

On 28.03.2013 author of the article filed a patent application with Ukrpatent for a new technical solution "Combined method of trichromatic brightness pyrometry". This invention [14] relates to the field of spectral pyrometry and can be used in the pyrometric determination of the actual temperature $\mathrm{T}$ of a selectively radiating surface with a known dispersion pattern, that is, the dependence of spectral emissivity on the wavelength: $\varepsilon=\mathrm{f}(\lambda)$ in the working range of the pyrometer $\lambda \in\left(\lambda_{1} \div \lambda_{3}\right)$.

The basis of the invention was the task of clarifying and expanding the functionality of the known method of trichromatic brightness pyrometry proposed by E.Payet.

The proposal is explained as follows: for the first time, the author here uses the expression not arithmetic average, but proportional average: $\varepsilon_{2}=\sqrt{\varepsilon_{1} \cdot \varepsilon_{3}}$. If we predict both sides of the expression $\varepsilon_{2}=\sqrt{\varepsilon_{1} \cdot \varepsilon_{3}}$, then we obtain the equality $\ln \varepsilon_{2}=$ $\left(\ln \varepsilon_{1}+\ln \varepsilon_{3}\right) / 2$. Substituting into it the values of the spectral emissivity, expressed from the brightness temperature formula: $\ln \varepsilon_{\mathrm{i}}=\left(\mathrm{C}_{2} / \lambda_{\mathrm{i}}\right) \cdot\left[\mathrm{T}^{-1}-\left(\mathrm{T}_{\mathrm{Si}}\right)^{-1}\right]$, eliminates three unknowns at once: $\varepsilon_{1}, \varepsilon_{2}$ and $\varepsilon_{3}$. As a result, only one unknown remains in the formula - the actual temperature $\mathrm{T}$, by describing the expression of which we get the following:

$$
\begin{aligned}
& \left(\mathrm{C}_{2} / \lambda_{2}\right) \cdot\left[\mathrm{T}^{-1}-\left(\mathrm{T}_{\mathrm{S} 2}\right)^{-1}\right]=\left\{\left(\mathrm{C}_{2} / \lambda_{1}\right) \cdot\left[\mathrm{T}^{-1}-\left(\mathrm{T}_{\mathrm{S} 1}\right)^{-1}\right]+\right. \\
& \left.\left(\mathrm{C}_{2} / \lambda_{3}\right) \cdot\left[\mathrm{T}^{-1}-\left(\mathrm{T}_{\mathrm{S} 3}\right)^{-1}\right]\right\} / 2 .
\end{aligned}
$$

Conducting transformations and summing up similar terms, we obtain an analytical solution of this equation for the actual temperature $\mathrm{T}$ in the form of a formula that has a very simple form:

$$
\begin{aligned}
\mathrm{T}=\mathrm{T}_{\mathrm{S} 2} \cdot\left\{1-1 / 2 \cdot \lambda_{2} \cdot\left[\left(\lambda_{1}\right)^{-1}+\right.\right. & \left.\left.\left(\lambda_{3}\right)^{-1}\right]\right\} /\left\{1-1 / 2 \cdot\left(\lambda_{2} \cdot \mathrm{T}_{\mathrm{S} 2}\right) \cdot\left[\left(\lambda_{1} \cdot \mathrm{T}_{\mathrm{S} 1}\right)^{-1}\right.\right. \\
& \left.\left.+\left(\lambda_{3} \cdot \mathrm{T}_{\mathrm{S} 3}\right)^{-1}\right]\right\},
\end{aligned}
$$

where $\mathrm{T}_{\mathrm{S} 1}, \mathrm{~T}_{\mathrm{S} 2}$ and $\mathrm{T}_{\mathrm{S} 3}$ are the brightness temperatures $(\mathrm{K})$ of of the selectively radiating surface, which are measured at wavelengths $\lambda_{1}, \lambda_{2}$ and $\lambda_{3}$ in the working range of the pyrometer $\lambda \in\left(\lambda_{1} \div \lambda_{3}\right)$.

\subsection{Determining the Intermediate Wavelength $\lambda_{2}$ for Brightness Pyrometry}

However, in order to obtain all the results presented above, it is necessary to choose the values of the intermediate wavelength $\lambda_{2}$ in such a way as to satisfy the equality $\varepsilon_{2}=$ $\sqrt{\varepsilon_{1} \cdot \varepsilon_{3}}$. This condition can be met mathematically rigorously - using the concept of the "inverse" function for $\lambda(\varepsilon): \lambda_{2}=$ $\mathrm{f}^{-1}\left(\varepsilon_{2}\right)=\mathrm{f}^{-1}\left(\sqrt{\varepsilon_{1} \cdot \varepsilon_{3}}\right) \equiv \mathrm{F}\left(\sqrt{f\left(\lambda_{1}\right) \cdot f\left(\lambda_{3}\right)}\right)$, i.e. for dispersion of spectral emissivity in the working range of the wave pyrometer $\lambda_{2}\left(\lambda_{1}<\lambda_{2}<\lambda_{3}\right)$.

As a result, the value of the intermediate wavelength depends only on the base wavelengths $\lambda_{1}$ and $\lambda_{2}$, as well as on the nature of the functional dependence $\varepsilon=f(\lambda)$ and does not depend on the absolute values of the spectral emissivity $\varepsilon_{1}, \varepsilon_{2}$ and $\varepsilon_{3}$. If the base values of the extreme wavelengths of the range of $\lambda_{1}$ and $\lambda_{3}$ are chosen based on the minimum of the instrumental error. For example, for a color pyrometer with a "vanishing filament", $\lambda_{1}=0.43 \mu \mathrm{m}, \lambda_{3}=0.65 \mu \mathrm{m}$. This is historically the first "blue-red" ratio of the wavelengths $\lambda_{1}$ and $\lambda_{3}$, which are the borders of the "sensitivity window" of the human eye. In this case, $\lambda_{2}$ is completely determined by the nature of the dispersion of the spectral emissivity $\varepsilon=f(\lambda)$ for any selective surface.

In cases where "transparency windows" are more important, for example, when absorption of water vapor in atmospheric air is very significant, other wavelengths are chosen.

EXAMPLE 1.

If the dependence character for the dispersion $\varepsilon=f(\lambda)$ of the radiating surface is a linear dependence $\varepsilon=a+b \lambda$, then the inverse function for $\varepsilon=\mathrm{f}(\lambda)$ has the following form: $\lambda=$ $(\varepsilon-a) / b$. Then $\lambda_{2}=\left(\varepsilon_{2}-a\right) / b$. Substituting instead of $\varepsilon_{2}$ its value $\varepsilon_{2}=\sqrt{\varepsilon_{1} \cdot \varepsilon_{3}}$, we get:

$$
\begin{aligned}
\lambda_{2}=\left(\sqrt{\varepsilon_{1} \cdot \varepsilon_{3}}-a\right) / b= & \left\{\sqrt{ }\left[\left(a+b \lambda_{1}\right) \cdot\left(a+b \lambda_{3}\right)\right]-a\right\} / b=\sqrt{ }[(a / b \\
& \left.\left.+\lambda_{1}\right) \cdot\left(a / b+\lambda_{3}\right)\right]-a / b .
\end{aligned}
$$

Thus, if it is known that the dispersion pattern of the spectral emissivity $\varepsilon=f(\lambda)$ the working range of the 
pyrometer has a linear form $\varepsilon=a+b \lambda$, then the temperature $\mathrm{T}_{\mathrm{S}}\left(\lambda_{2}\right)$ should be measured at an intermediate wavelength $\lambda_{2}$ $\left(\lambda_{1}<\lambda_{2}<\lambda_{3}\right)$, which is determined from the nonlinear expression of the base wavelengths at the boundaries of the working range of the pyrometer $\lambda_{1}, \lambda_{3}$ and of the characteristic wavelength $\lambda_{\mathrm{o}}=a / b$. This expression $\lambda_{2}$ depends only on the nature of the dispersion $\varepsilon(\lambda)$, and does not contain the absolute values of the emissivity of the surface:

$$
\lambda_{2}=\sqrt{ }\left[\left(\lambda_{\mathrm{o}}+\lambda_{1}\right) \cdot\left(\lambda_{\mathrm{o}}+\lambda_{3}\right)\right]-\lambda_{\mathrm{o}} .
$$

Since the value of $\lambda_{\mathrm{o}}$ can take both positive and negative values, it is better to rewrite this formula in this form:

$$
\lambda_{2}=\sqrt{ }\left[\left(\lambda_{1}+\lambda_{\mathrm{o}}\right) \cdot\left(\lambda_{3}+\lambda_{\mathrm{o}}\right)\right]-\lambda_{\mathrm{o}}
$$

It should be noted here that in the proposed method it is necessary to have a priori not absolute values of spectral emissivity: $\boldsymbol{\varepsilon}_{1,} \boldsymbol{\varepsilon}_{2}$ and $\boldsymbol{\varepsilon}_{3}$, but only the nature of the dispersion of spectral emissivity $\varepsilon=f(\lambda)$, which is given by the ratio $(a / b)$ for selective surface in the working range of the pyrometer $\lambda \in\left(\lambda_{1} \div \lambda_{3}\right)$.

EXAMPLE 2 .

If the dependence character $\varepsilon=\mathrm{f}(\lambda)$ is expressed by the formula $\ln \varepsilon=a+b \lambda$, then the inverse function for $\varepsilon=\mathrm{f}(\lambda)$ is expressed as follows $\lambda=(\ln \varepsilon-a) / b$. Then $\lambda_{2}=\left(\ln \varepsilon_{2}-a\right) / b$. Substituting instead of $\varepsilon_{2}$ its value of $\varepsilon_{2}=\sqrt{ }\left(\varepsilon_{1} \cdot \varepsilon_{3}\right)$, we get: $\lambda_{2}$ $=\left[\ln \sqrt{ }\left(\varepsilon_{1} \cdot \varepsilon_{3}\right)-a\right] / b=\left[\left(\ln \varepsilon_{1}+\ln \varepsilon_{3}\right) / 2-a\right] / b$. And replacing $\ln \varepsilon_{1}$ and $\ln \varepsilon_{3}$ with their expressions, we get the result:

$$
\begin{aligned}
\lambda_{2}= & {\left[\left\{\left(a+b \lambda_{1}\right)+\left(a+b \lambda_{3}\right)\right\} / b-a\right] / b=\left[\left\{2 a+b\left(\lambda_{1}+\lambda_{3}\right)\right\} /\right.} \\
& 2-a] / b=\left(\lambda_{1}+\lambda_{3}\right) / 2 .
\end{aligned}
$$

Thus, if it is known a priori that the dispersion pattern of the surface spectral emissivity $\varepsilon=\mathrm{f}(\lambda)$ in the working range of the pyrometer has the form $\ln \varepsilon=a+b \lambda$, then the brightness temperature $\mathrm{T}_{\mathrm{S}}\left(\lambda_{2}\right)$ should be measured at an intermediate wavelength $\lambda_{2}\left(\lambda_{1}<\lambda_{2}<\lambda_{3}\right)$, which is equal to the arithmetic average of the base wavelengths at the borders of the working range:

$$
\lambda_{2}=\left(\lambda_{1}+\lambda_{3}\right) / 2
$$

For the case of $\lambda_{1}=0.43 \mu \mathrm{m}, \lambda_{3}=0.65 \mu \mathrm{m}$, we get $\lambda_{2}=$ $0.540 \mu \mathrm{m}$.

EXAMPLE 3.

Since the dependence character $\varepsilon=\mathrm{f}(\lambda)$ for metals (Drude formula) has the form $\varepsilon=0.365 \sqrt{ }(\mathrm{r} / \lambda)$, the inverse function for $\varepsilon=\mathrm{f}(\lambda)$ is as follows $\lambda=\mathrm{r} \cdot(0.365 / \varepsilon)^{2}$. Then $\lambda_{2}=$ $\mathrm{r} \cdot\left(0.365 / \varepsilon_{2}\right)^{2}$. Substituting instead of $\varepsilon_{2}$ its value of $\varepsilon_{2}=$ $\sqrt{\varepsilon_{1} \cdot \varepsilon_{3}}$, we get:

$$
\begin{aligned}
\lambda_{2}=0,365^{2} \cdot \mathrm{r} /\left(\varepsilon_{1} \cdot \varepsilon_{3}\right)= & 0,365^{2} \cdot \mathrm{r} /\left[0,365 \sqrt{ }\left(\mathrm{r} / \lambda_{1}\right)\right] \cdot\left[0,365 \sqrt{ }\left(\mathrm{r} / \lambda_{3}\right)\right]= \\
& \sqrt{\lambda_{1} \cdot \lambda_{3}} .
\end{aligned}
$$

Since it is known a priori that for a metal surface the dispersion pattern of the spectral emissivity $\varepsilon=f(\lambda)$ in the working range of the pyrometer has the form $\varepsilon=0.365 \sqrt{ }(\mathrm{r} / \lambda)$, the brightness temperature $\mathrm{T}_{\mathrm{S}}\left(\lambda_{2}\right)$ must be measured at an intermediate wavelength $\lambda_{2}\left(\lambda_{1}<\lambda_{2}<\lambda_{3}\right)$, which is equal to the average proportional to the base wavelengths at the boundaries of the working range of the pyrometer:

$$
\lambda_{2}=\sqrt{\lambda_{1} \cdot \lambda_{3}} .
$$

For the case of $\lambda_{1}=0.43 \mu \mathrm{m}, \lambda_{3}=0.65 \mu \mathrm{m}$, we get $\lambda_{2}=$ $0.528 \mu \mathrm{m}$.

\section{Findings}

The article is based on the analysis of two main methods of spectral pyrometry (color and brightness) within the framework of the trichromatic method, i.e. on the basis of three measurements of the brightness temperature $T_{S}$, analytical expressions for the actual temperature $\mathrm{T}(\mathrm{K})$ of the surface were obtained for the first time, and explicitly for the brightness method.

The expression "average proportional" $\varepsilon_{2}=\sqrt{\varepsilon_{1} \cdot \varepsilon_{3}}$ was used for the first time as the "equation of coupling" for the method of brightness pyrometry. And in the method of color pyrometry (for a double spectral ratio), the expression "arithmetic mean" $\varepsilon_{2}=\left(\varepsilon_{1}+\varepsilon_{3}\right) / 2$ was used.

For the first time, to determine the intermediate ("effective") wavelength in the trichromatic method of spectral pyrometry, the "inverse function" $\lambda=\mathrm{f}^{-1}(\varepsilon)$ option was used to express the dispersion of surface emissivity.

It turned out that for determining the actual temperature $\mathrm{T}$ $(\mathrm{K})$ of a selective surface, it is not the values of emissivity $\varepsilon$ that are important, but only the nature of the dispersion, i.e. the type of the function $\varepsilon=f(\lambda)$ (linear, logarithmic, etc.), since the use of the "inverse function" option allows for any pre-specified dispersion character to obtain an expression for $\lambda=\mathrm{f}^{-1}(\varepsilon)$ and determine value $\lambda_{2}$.

The main disadvantage of the spectral methods of pyrometric determination of temperatures is the need to have a priori the nature of the dispersion $\varepsilon=f(\lambda)$ for a radiating selective surface. Therefore, the most promising method of pyrometric temperature measurement for cases where the nature of the dispersion is unknown is the combined approach of the author $[15,16]$, using a combination of both pyrometry methods: spectral and radiation.

\section{Acknowledgements}

The author is grateful to Alexander Magunov, a wellknown Russian specialist in the field of pyrometry, for a valuable discussion, as well as for an invaluable gift - the monograph "Spectral Pyrometry" (with a dedication).

\section{References}

[1] Ribot G. Optical pyrometry. M. L.: GTTI, 1934.

[2] Gordov A. N. Fundamentals of pyrometry. M.: Metallurgy, 1971. 
[3] Ludanov K. I. UA Patent No.63080 IPC G01J 5/00 Combined Method of Radiation Pyrometry. 15.01.2004, Bul. №1.

[4] Ludanov K. I. UA Patent No. 107108 IPC G01J 5/00 Combined Method of Partly Radiation Pyrometry 25.11.2014, Bul. №22.

[5] Ludanov K. I. Combined Method of Radiation Pyrometry in Material Science // Inter. Journal IJLRET, Vol. 04 - Issue 05 // May 2018 // PP. 01-06.

[6] Temperature measurements. DIRECTORY. Kiev: "Naukova Dumka", 1989.

[7] Bloch A. Fundamentals of heat transfer by radiation. M.; 1961.

[8] Swet D. Ya. Optical methods for measuring true temperatures. M.: "Science", 1982.

[9] Magunov A. N. Spectral Pyrometry. M.: "Fizmatlit", 2012.

[10] Pyatt E. C. Brit. J. Appl. Phys., Vol. 5, p. 264.

[11] Svet D. Ya. USSR certificate of authorship, № 476464, 1975.
[12] Ludanov K. I. UA Patent No.73231 IPC G01J 5/00 Combined method of trichromatic color pyrometry. 06.15.2005, Bul. №6.

[13] Vilenkin $N . Y$. The method of successive approximations. M: "Fizmatgiz", 1961.

[14] Ludanov K. I. UA Patent No.82870 IPC G01J 5/00 Combined method of trichromatic brightness pyrometry. 08.27.2013, Bul. №16.

[15] Ludanov K. I. UA Patent No.104511 IPC G01J 5/00 Pyrometric Method for the Joint Determination of the Absolute Temperature and the Radiation Capacity of a Surface. 10.02.2014, Bul. №3.

[16] Ludanov K. I. WO 2014/046638 IPC G01J 5/00 Pyrometric Method for the Joint Determination of the Actual Temperature and the Radiation Capacity of a Surface. PCT/UA2013/000087, 12.08.2013. 